\title{
Breast Cancer Disparities Through the Lens of the COVID-19 Pandemic
}

\author{
Lisa Newman ${ }^{1} \cdot$ Laura Fejerman $^{2} \cdot$ Tuya Pal $^{3} \cdot$ Eralda Mema $^{4} \cdot$ Geraldine McGinty $^{4}$ - Alex Cheng ${ }^{5} \cdot$ Mia Levy $^{5}$. \\ Adeyiza Momoh ${ }^{6} \cdot$ Melissa Troester $^{7} \cdot$ Bryan Schneider $^{8} \cdot$ Lorna McNeil $^{9} \cdot$ Melissa Davis $^{1} \cdot$ Kemi Babagbemi $^{4}$. \\ Kelly Hunt ${ }^{10}$
}

Accepted: 19 May 2021 / Published online: 6 August 2021

(C) The Author(s), under exclusive licence to Springer Science+Business Media, LLC, part of Springer Nature 2021

\begin{abstract}
Purpose of Review The emergency medicine and critical care needs of the COVID-19 pandemic forced a sudden and dramatic disruption of cancer screening and treatment programs in the USA during the winter and spring of 2020. This review commentary addresses the impact of the pandemic on racial/ethnic minorities such as African Americans and Hispanic-Latina Americans, with a focus on factors related to breast cancer.

Recent Findings African Americans and Hispanic-Latina Americans experienced disproportionately higher morbidity and mortality from COVID-19; many of the same socioeconomic and tumor biology/genetic factors that explain breast cancer disparities are likely to account for COVID-19 outcome disparities.

Summary The breast cancer clinical and research community should partner with public health experts to ensure participation of diverse patients in COVID-19 treatment trials and vaccine programs and to overcome COVID-19-related breast health management delays that are likely to have been magnified among African Americans and Hispanic-Latina Americans.
\end{abstract}

Keywords Breast cancer · Disparities · COVID-19 · Clinical research · African Americans · Hispanic/Latina Americans

Disparities in the impact of the COVID-19 pandemic related to race/ethnicity and poverty were predictable. The tragedy of healthcare access barriers surrounding any socioeconomically disadvantaged community results in higher morbidity and mortality from a spectrum of diseases such as hypertension, diabetes, obesity, and cancer. Rates of poverty and unemployment are disproportionately high among African Americans and Hispanic/Latina Americans compared to non-Hispanic Whites; consequently, these population subsets also experience a disproportionately high burden of both malignant and benign diseases $[1,2]$. Not surprisingly, mortality from COVID-19 has now been added to this list, and death rates from this pandemic are significantly higher among these racial-ethnic minority population subsets [3].

This article summarizes components of the Topical Collection on Breast Cancer Disparities found in this link: https://link.springer.com/journal/ 12609/topicalCollection/AC_62d360fce215be55d03b0430e628f389/page/1.

Lisa Newman

lan4002@med.cornell.edu

Extended author information available on the last page of the article
The severity of COVID-19 in African American and Hispanic Latina communities has been especially harsh because of various features related to population demographics and the epidemiology of contagious diseases.

(i) Urban areas are the epicenters of the COVID-19 pandemic, and racial-ethnic minorities are more heavily concentrated within urban cities in the USA.

(ii) Lower income apartment complexes that house disproportionately higher numbers of racial-ethnic minorities are poorly suited to optimal social distancing recommendations.

(iii) Racial-ethnic minorities account for a disproportionately high number of "essential worker" jobs that are exempt from shelter-in-place mandates, such as healthcare facilities buildings and maintenance staff, delivery services, and public transportation services. COVID-19 exposures are magnified in these environments resulting in greater risks for the workers as well as individuals with whom the workers share a household.

(iv) Racial-ethnic minorities in the essential services workforce are more likely to utilize public transportation, 
where social distancing is more difficult and COVID-19 exposures are increased.

(v) Racial-ethnic minorities are more likely to reside in multi-generational households, resulting in the more vulnerable elders in these families facing increased exposures.

Several other downstream consequences of the COVID-19 pandemic are also predictable but the oncology community is now facing a narrow window of opportunity to utilize lessons that we have learned from breast cancer disparities to work proactively in mitigating these adverse outcomes before they become reality. Herein, we present a series of approaches where the breast cancer disparities research community can partner with the infectious disease/public health community in preventing one public health crisis from precipitating several others:

Ensuring accrual of diverse patients onto COVID-19related clinical trials and participation in vaccine programs: The breast cancer clinical trials community has developed powerful networks involving advocates, navigators, and community engagement programs to improve the diversity of patients participating in prevention, research, and treatment trials. While accrual diversity has certainly not yet been optimized in cancer clinical research, the power of these networks can and should be leveraged for the benefit of COVID-19 vaccine programs and treatment trials. It is imperative that COVID-19 clinical research studies and vaccination programs be designed so that they reach racial-ethnic minorities. Statistical sampling and accrual targets should specifically address the importance of racial-ethnic minority accrual, and oversampling efforts to achieve these targets should be built into the budgets for these studies. As described by Troester et al., oversampling of African Americans was a key strategy for generating meaningful information regarding disparities from the Carolina Breast Cancer Study. McNeil et al. discuss the value of community engagement efforts in clinical research, and the Sisters Network, Inc ${ }^{\circledR}$ present unique outreach approaches that patient advocates can implement.

Lessening breast cancer stage migration following COVID-19-related hiatus in early detection programs: Mammography screening trials have largely been placed on hiatus during the COVID-19 pandemic, as healthcare systems have had to respect social distancing/shelter-in-place efforts of both patients and facility personnel. Racial-ethnic minority population subsets already have more advanced stage distribution of breast cancer, which clearly contributes to their disproportionate mortality burden. The breast oncology community must work aggressively with public health systems to restore and strengthen screening programs in underserved communities. Bagbagbemi et al. discuss the importance of screening mammography in achieving breast health equity.
Supporting the safety net public hospitals: Public hospitals in the urban pandemic epicenters serve as the safety nets of health care for medically underserved and socioeconomically disadvantaged communities; these institutions have therefore been disproportionately devastated by the financial costs of treating COVID-19 pneumonia and COVID-19-related multi-system organ failure. These same safety net healthcare facilities also provide cancer care to a disproportionately high volume of racial-ethnic minority patients. The oncology community must work with public health advocates to support legislation that will restore the financial solvency of safety net hospitals so that the cancer detection and treatment needs of their patients will continue to be met. Many of these public hospitals can serve as the sources of "big data"; utilization of data generated by large health systems and artificial intelligence approaches are discussed by Levy and Cheng, and Mema and McGinty.

Incorporating disparity-related research questions into COVID-19 clinical and translational research studies: Breast cancer disparities associated with race-ethnicity are increasingly being defined in the context of cancer pathogenesis pathways that are linked to ancestral genetics. Some of these ancestrally defined genetics are also linked to immune response pathways and are therefore likely to be relevant to infectious disease outcomes. COVID-19 translational research and correlative science studies should therefore be designed a priori to include questions related to the ancestral genetic components of race-ethnicity. Davis and Newman, and Fejerman et al. discuss the genetics of African and Hispanic/Latina ancestry, respectively, and Schneider et al. discuss the role of African ancestry in toxicity of disease treatment. Reid and Pal discuss the importance of studying germline genetics in diverse populations.

Addressing the impact of COVID-19 on the economy and health equity efforts: Social distancing and shelter-in-place mandates have been the cornerstone of public health efforts to control the COVID-19 pandemic. Tragically, these efforts have also led to massive rates of unemployment and loss of healthcare insurance. Racial-ethnic minorities such as African Americans and Hispanic-Latina Americans have historically been disproportionately represented among the unemployed and are likely to be similarly over-represented among the newly unemployed and newly noninsured or under-insured population resulting from the COVID-19 impact on the economy. The oncology community must work in partnership with public health advocates in supporting legislation that broadens access to healthcare coverage for these additional victims of the pandemic. Momoh et al. discuss the implications of health insurance on access to surgical cancer care.

It is clear that COVID-19 will have unprecedented downstream effects on overall public health and on health disparities in particular. The breast cancer disparities research community has been studying and implementing approaches to 
achieve health equity for many decades. By working collaboratively and proactively, we can pre-empt several of the anticipated pandemic sequelae.

\section{References}

1. Brawley OW, Berger MZ. Cancer and disparities in health: perspectives on health statistics and research questions. Cancer. 2008;113(7 Suppl):1744-54.
2. Newman LA. Breast cancer disparities: socioeconomic factors versus biology. Ann Surg Oncol. 2017;24:2869-75.

3. Webb Hooper M, Napoles AM, Perez-Stable EJ. COVID-19 and racial/ethnic disparities. JAMA. 2020;323:2466-7.

Publisher's Note Springer Nature remains neutral with regard to jurisdictional claims in published maps and institutional affiliations.

\section{Affiliations}

\section{Lisa Newman ${ }^{1} \cdot$ Laura Fejerman $^{2} \cdot$ Tuya Pal $^{3} \cdot$ Eralda Mema $^{4} \cdot$ Geraldine McGinty $^{4} \cdot$ Alex Cheng $^{5} \cdot$ Mia Levy $^{5}$. Adeyiza Momoh ${ }^{6} \cdot$ Melissa Troester $^{7} \cdot$ Bryan Schneider $^{8} \cdot$ Lorna McNeil $^{9} \cdot$ Melissa Davis $^{1} \cdot$ Kemi Babagbemi $^{4}$. Kelly Hunt ${ }^{10}$}

1 Department of Surgery, Weill Cornell Medicine, New York, NY, USA

2 Department of Public Health Sciences, University of California Davis Health, Sacramento, CA, USA

3 Department of Medicine, Vanderbilt-Ingram Cancer Center, Nashville, TN, USA

4 Department of Radiology, Weill Cornell Medicine, New York, NY, USA

5 Department of Biomedical Informatics, Vanderbilt University, Nashville, TN, USA
6 Department of Surgery, University of Michigan, Ann Arbor, MI, USA

7 Department of Epidemiology, University of North Carolina, Chapel Hill, NC, USA

8 Department of Medicine, Indiana University, Indianapolis, IN, USA

9 Department of Health Disparities, University of Texas M.D. Anderson Cancer Center, Houston, TX, USA

10 Department of Breast Surgery, University of Texas M.D. Anderson Cancer Center, Houston, TX, USA 